\title{
Mental Health Strategies to Combat the Psychological Impact of Coronavirus Disease 2019 (COVID-19) Beyond Paranoia and Panic
}

Cyrus SH $\underline{\mathrm{Ho}},{ }^{1} M B B S, M R C P s y c h, F A M S$, Cornelia YI $\underline{\text { Chee, }},{ }^{1} M B B S$, MMed (Psychiatry), Roger CM $\underline{\text { Ho }},{ }^{2,3}{ }_{F R C P s y c h, ~ F R C P C, F A M S}$

On 30 January 2020, the World Health Organization (WHO) declared the outbreak of coronavirus disease 2019 (COVID-19) an international public health emergency after the number of cases soared across 34 regions in Mainland China and surpassed that of severe acute respiratory syndrome (SARS) in 2003. The virus was believed to have originated from a wholesale seafood market in the city of Wuhan in the province of Hubei towards the end of December 2019. Shortly after, the number of cases increased exponentially in Wuhan and nearby cities and provinces before spreading throughout the world.

Located approximately $3432 \mathrm{~km}$ from the epicentre of Wuhan, Singapore is a densely populated city-state of 5.7 million who saw 1,592,612 international visitors in 2019; of these, 380,933 were visitors from Mainland China. ${ }^{1}$ After a tourist from Wuhan was identified as the first case of COVID-19 infection on 23 January 2020 in Singapore, the country responded decisively by initiating a series of public health measures to contain the outbreak that included travel advisories, restriction of entry into the country by individuals who had travelled to Mainland China in the preceding 2 weeks, mandatory quarantine for contact cases and rigorous contact tracing of individuals linked to confirmed COVID-19 cases.

On 7 February 2020, Singapore raised the Disease Outbreak Response System Condition (DORSCON) - a colour-coded framework that maps the current disease situation in the country-from Yellow to Orange after there was confirmatory evidence of community transmission of the virus involving several confirmed COVID-19 patients who were not linked to any existing cases and had no travel history to China. At the Orange level, the outbreak is deemed to have moderate to high impact on the health of the public. The last time it was raised to Orange was during the $\mathrm{H} 1 \mathrm{~N} 1$ flu pandemic in 2009, and would also have been the case during the SARS outbreak in 2003 had it been in place.
After DORSCON was raised to Orange, it triggered off-on the same day-widespread panic buying of food items and toiletries across the country, leading many stores to run out of supplies at short notice. This phenomenon was attributed to the intentions of locals who wanted to stock up on groceries after they feared exposure to heightened viral transmission. Additionally, the Ministerial Task Force that was convened to manage the COVID-19 outbreak had suggested the country needed to be psychologically prepared for the fallout from the current outbreak to be worse than the 2003 SARS crisis. The magnitude of fear and uncertainty among the public was so excessive that it prompted the Prime Minister of Singapore to address the public and reassure them that the country has adequate food supplies, while at the same time urging calm and prudence with their purchases. The response of locals to the pandemic, which has been likened to mass hysteria and paranoia, has led many to question their mental health and resilience.

More than a month into the current pandemic, 77,816 people from around the world have been infected as of 22 February 2020, of which 21,147 have recovered from the illness and 2360 have died. ${ }^{2}$ Outside Mainland China, 32 countries and territories were affected, with Singapore ranked third as having the most number of confirmed COVID-19 cases after South Korea and Japan. Of the 86 cases that were tested positive for the virus with real-time reverse transcriptase-polymerase chain reaction, 47 patients had recovered and been discharged. ${ }^{3}$

In an infectious disease outbreak, the mounting fear that is aroused in individuals is a common phenomenon and can lead to erratic behaviour in them. It can afflict anyone irrespective of gender and sociodemographic status. This is true of COVID-19, especially when there is still much speculation surrounding its mode of transmission and the disease is spreading at an unparalleled rate with no definitive treatment in sight. In the early days of the COVID-19 outbreak in Mainland China, a survey

\footnotetext{
${ }^{1}$ Department of Psychological Medicine, University Medicine Cluster, National University Health System, Singapore

${ }^{2}$ Department of Psychological Medicine, National University of Singapore, Singapore

${ }^{3}$ Health Innovation and Technology (iHealthtech), National University of Singapore, Singapore

Address for Correspondence: Dr Cyrus Ho Su Hui, Department of Psychological Medicine, University Medicine Cluster, National University Health System, Level 9, NUHS Tower Block, 1E Kent Ridge Road, Singapore 119228.

Email: su_hui_ho@nuhs.edu.sg
} 
found that $53.8 \%$ of respondents rated the psychological impact of the outbreak as moderate or severe, $16.5 \%$ reported moderate to severe depressive symptoms, $28.8 \%$ reported moderate to severe anxiety symptoms, and $8.1 \%$ reported moderate to severe stress levels. ${ }^{4}$ Compared to the SARS outbreak 17 years ago, the fear among the public was even more palpable as increased air travel and entrenched global connectedness made the spread of COVID-19 appeared much more rampant. Extensive coverage of the pandemic by the mass media also influences the physical and psychological response of the public to the infectious disease threat, which may amplify their apprehension even when it is used to encourage them to take precautionary and preventive measures to protect themselves from the virus. ${ }^{5,6}$

Research has demonstrated the wide and profound psychological impact outbreaks can have on people. For individuals without mental illness, an outbreak can induce psychiatric symptoms; in those with pre-existing mental illnesses, their conditions could be aggravated and cause distress to their caregivers. Regardless of exposure, individuals may experience fear and anxiety of falling ill or dying, helplessness or blame those who are ill, which can potentially trigger off a mental breakdown. ${ }^{7}$ Significant psychiatric morbidities have been found to vary from anxiety, depression, panic attacks, somatic symptoms and post-traumatic stress disorder (PTSD) to delirium, psychosis and even suicidality, ${ }^{7-9}$ and have been associated with younger age and increased self-blame. ${ }^{10}$

In those who are grieving the sudden and traumatic loss of loved ones from the current outbreak, an inability to gain closure can lead to anger and resentment. ${ }^{11}$ In patients and those who are quarantined, they may experience guilt, shame or stigma. Studies have reported high prevalence of psychological distress in those who undergo a longer period of quarantine, and it is also associated with increased PTSD prevalence that is correlated with depressive symptoms. ${ }^{12}$ In the community, there is distrust of others in terms of disease spread and the capability of the authorities and health services to contain the outbreak. With cessation of community services and collapse of work industries that impact adversely on the economy, many individuals incur financial losses and run the risk of unemployment, further intensifying the negative emotions experienced by them. ${ }^{13}$

At the international level, communities that have been affected by the outbreak may be targeted for blame and stigma by other countries over fear of infection and this may impede cross-national trade and fuel further unrest. Since emotions can be amplified by pre-existing depressive and anxiety disorders and lead to increased rumination on contracting the virus, the behaviour and social interactions of individuals with others can be profoundly remodelled.

Psychological responses are associated with particular health-seeking behaviours. In a community survey of uninfected individuals during the SARS crisis in Hong Kong, it was found that those with a moderate level of anxiety and stronger perception of risk of contracting SARS were more likely to take comprehensive, precautionary measures to protect themselves from the virus. ${ }^{14}$ Nonetheless, the feelings of helplessness and anxiety can often motivate individuals to resort to the use of unproven methods and remedies that may prove detrimental to their health.

Medical responders-including first responders such as paramedics and ambulance personnel—and healthcare workers (HCWs) have been found to display heightened stress and become emotionally affected and traumatised, and they also experience higher levels of anxiety and depression. ${ }^{15}$ This is understandable since the anxiety and fear of becoming infected is higher from greater risk of increased exposure. There is also fear of infecting their loved ones and children. The delicate balance between the call of professional duty, altruism and fear for oneself and others often causes conflict and dissonance in many HCWs. ${ }^{16}$

Studies have shown that HCWs who work in emergency departments, intensive care units and isolation wards have a greater risk of developing adverse psychiatric outcomes than those from other job departments. This could be attributed to their direct exposure to infected patients and the demanding nature of their work. ${ }^{17} \mathrm{~A}$ study from Singapore reported that doctors and those who were single were at a higher risk of developing psychiatric symptoms than nurses and those who were married. ${ }^{18}$ A systematic review of the impact a disaster can have on the mental health of HCWs has identified common risk factors for the development of psychological morbidities that include lack of social support and communication, maladaptive coping and lack of training. ${ }^{17}$

During pandemics, the focus of public health authorities and the mass media is on biological and physical repercussions of the outbreak than on mental health issues. However, with a growing number of reports on the increasing mental health burden caused by the COVID-19 outbreak, there have been more calls for measures to enhance mental health support for the public. On 27 January 2020, the National Health Commission in Mainland China issued the first comprehensive guidelines on emergency psychological crisis intervention in individuals who were affected 
by COVID-19; ${ }^{19}$ the emphasis was on delivery of mental health support services to patients and HCW by multidisciplinary teams that consisted of mental health professionals.

In Singapore, psychological defence is one of 5 pillars in her Total Defence strategy to maintain faith and trust between the population and her government during a national crisis and to shore up resilience. Throughout this pandemic, the government and its health authority, the Ministry of Health, have kept the public abreast on the progress of the outbreak with regular news broadcasts and announcements on social media. These include daily updates such as the number of new and current infections, patients who are in critical condition or have been discharged and preventive measures. Social media channels have also been set up by the state to curb the spread of false information and "fake news". Regular dialogue with Cabinet Ministers and infectious diseases physicians is aired to clear doubts.

Before the current pandemic, Singapore has an established network of mental health services to meet the mental health needs of her population. They range from psychiatric clinics in all public hospitals and some polyclinics to private psychiatric and psychotherapy clinics and family service centres. Nevertheless, there are no national guidelines to support the mental health of the nation during the COVID-19 outbreak. To strengthen the mental health strategy of the country, 6 critical areas are identified that involve psychiatrists in specific roles. The following discussion will also help incumbent governments, hospitals and communities overseas to manage mass hysteria and paranoia that follows an outbreak and after viral transmission has occurred in the community.

\section{Identification of High-Risk Groups}

Health authorities must identify vulnerable groups in the community who are at high risk of psychological morbidities and target them for early psychological interventions. Additionally, foreigners who have been quarantined or isolated in hospitals are at increased risk of psychiatric episodes since they have been deprived of social support and face the risk of being repatriated back to their countries of origin. Consequently, they will benefit from practical and emotional support. Although there is a proliferation of medical studies on COVID-19, very few have examined the psychological impact the disease has on individuals.

In their study of 1210 Chinese residents in the 2 weeks that followed the outbreak of COVID-19 in Mainland China, Wang et al found that women reported higher levels of anxiety, depression and stress. ${ }^{4}$ Their finding corroborated previous epidemiological studies that found women have an elevated risk of depression ${ }^{20}$ which could be attributed to their unique biology and socioeconomic factors. ${ }^{21}$ Although Wang et al also highlighted that students suffered greater psychological distress, this finding could be attributed to the closure of schools in Mainland China for an indefinite period and might not be generalisable to Singapore.

Nevertheless, should the situation in Singapore deteriorate and necessitate the closure of schools, the mental well-being of students in the country would merit study. Consequently, it is important that psychiatrists and public health experts undertake local epidemiological research so that their findings can provide a basis to introduce appropriate and targeted interventions.

\section{Improved Screening of Psychiatric Morbidities}

The finite number of mental health professionals in the country has made it essential that all physicians, particularly family and Emergency Department physicians, proactively screen patients for psychological issues when the latter visit them. The study by Wang et al had found that patients who presented with physical symptoms such as chills, coryza, cough, dizziness, myalgia and sore throat, those who rated their own health as poor and had a history of chronic illnesses were correlated with higher levels of anxiety, depression and stress that were attributed to the psychological impact of the outbreak. ${ }^{4}$ This is understandable since the symptoms of COVID-19 are non-specific and difficult to distinguish from other viral illnesses. ${ }^{22}$

In the early stages of the disease, little is known about the characteristics of the virus in terms of its mode of transmission, virulence and transmissibility. This lack of understanding has fuelled further anxiety and uncertainty. It is therefore necessary to screen individuals for any history of psychiatric disorders and whether they have young children. This is because the psychological health of parents may be affected when they become fearful of the risk of infecting their own children.

To aid them in evaluating the mental state of their patients and those who are under quarantine, health professionals can consider the use of standard instruments such as the Impact of Event Scale-Revised that was used during public health crises in Singapore in the past. ${ }^{10,23}$ They can also leverage on smartphone technology to do so. ${ }^{24}$ Physicians can make use of the opportunity to provide patients with resources on psychological support and, when needed, refer them to psychiatrists for further evaluation and management. 


\section{Mode and Content of Psychological Intervention}

In their efforts to curb the spread of COVID-19 that may result from face-to-face contact and therapy, several hospitals have launched online psychotherapy to manage psychiatric patients on video conferencing platforms such as Zoom. To address the needs of the general population during this pandemic, it is worthwhile to contemplate the introduction of online or smartphonebased psychoeducation on the outbreak to promote mental wellness and psychological interventions such as cognitive behavioural therapy (CBT) and mindfulnessbased cognitive therapy (MBCT).

In patients who exaggerate the risk of contracting and dying from COVID-19, CBT may challenge their cognitive biases. Although behavioural therapy can help them to combat anxiety with the use of relaxation techniques and prevent depression onset by altering the schedule of their routine activities, CBT can mitigate maladaptive coping behaviours such as avoidance, antagonistic confrontation and self-blame by enhancing their ability to manage stress. Maladaptive coping behaviours are associated with worse psychological outcomes. ${ }^{10,25}$

MBCT, which focuses on the use of various mindfulness meditation practices to cultivate nonjudgemental awareness in the present, have been found to be particularly helpful in alleviating stress in people with physical conditions. ${ }^{26}$ When it is hosted on virtual platforms, MBCT can benefit patients who are infected and nursed in isolation rooms as well as those who are quarantined at home with no access to mental health professionals. Online platforms could also be a means for individuals to provide peer support to each other and to share their challenges and resolutions during the outbreak to foster comradeship and resilience in them.

\section{More Support for Frontline Health Workers}

It is important to safeguard the morale and mental health of HCWs since they can impact the outcome and success of delivery of health services. ${ }^{27}$ Health facilities may consider shorter work hours, regular rest periods and rotating shifts for staff who work in high-risk jobs. It has been found that support from colleagues and supervisors and clear communication of directives and precautionary measures can reduce psychiatric symptoms ${ }^{18}$ Confidence in infection control measures may also mitigate and facilitate adaptive stress response ${ }^{28}$ Consequently, it is imperative to provide staff with adequate training on infection control. Hospital directives and protocols on COVID-19 should be clear, precise and disseminated to all staff.
Preventive measures must be put in place to ensure that HCWs do not become infected with the virus while at work. When they are infected, such incidents should be treated as work-related injuries. Their superiors can make a conscious effort to support staff and to set up a peer support system. It is vital to identify staff who suffers from work exhaustion or psychological distress so that timely intervention can be provided to them, and they should be encouraged to report their condition or mental state without fear of being blamed for doing so.

\section{Accurate Dissemination of Health and Related Information to the Public}

To minimise the detrimental impact of "fake news" that is so rampant in the social media, the government and health authorities must relay to the public timely and accurate evidence-based information on the pandemic through traditional and new media. Practical tips on how the public should react during the outbreak - such as the practice of good hygiene and donning of surgical maskand manage fear and uncertainty of the virus - through positive reframing, stress management and relaxation techniques - can be disseminated to the public through video clips and cartoons that can be easily understood by them. Higher levels of satisfaction with existing health information have been found to correlate with lower psychological distress in individuals.

Accurate and updated information on the number of recovered cases, treatment (such as medicine or vaccine) and mode of transmission as well as regualr updates on the number of infected cases and localities (such as real-time or virtual map) are associated with lower stress and anxiety, respectively. ${ }^{4}$ When individuals have access to adequate information and have sufficient trust in the government and health authorities to manage COVID-19, this could potentially reduce their anxiety and perceived vulnerability to the virus. ${ }^{29}$ With growing confidence in the measures introduced by the authorities, there is better adherence to precautionary and preventive measures that will encourage the wider community to work together to combat the outbreak.

The government, community leaders and health facilities also play a vital role to maintain racial harmony to prevent discrimination and stigma that accompany an outbreak..$^{30}$ In the current pandemic, there are reports of xenophobic attacks against individuals of Asian descent that included refusals to be seated next to them on public transport, refused entry to restaurants, verbal attacks on social media and even physical assaults. In response, the WHO and the Centers for Disease Control in the United States have issued official statements and pamphlets that condemned such actions and behaviours. 
Hopefully, with continued education on COVID-19 and constant reiteration to the public that viruses have no respect for borders, their fear of the unknown and magnitude of discrimination against other ethnicities can be curtailed.

\section{Integration of Hospital and Community Resources}

In Singapore, community psychiatric partners such as the Social Service Agencies (SSA) form an important first line to provide counselling to members of the public who need it during the current outbreak. In doing so, it strengthens mental resilience in the community and reduces the likelihood of psychiatric morbidities developing in individuals. Silver Ribbon (Singapore) and Fei Yue Community Services also provide online counselling and emotional support on COVID-related issues. Finally, a group of psychologists from the Singapore Psychological Society have been providing their services pro bono or at reduced rates to those who have been distressed by the outbreak.

In hospitals, psychiatrists have been providing additional clinic sessions to render psychiatric support to patients with emotional issues coming through the Emergency Departments. Nevertheless, there is still a need to combine resources to provide a comprehensive and integrated psychological service for patients and to enhance the psychological preparedness of the nation.

\section{Conclusion}

In the current pandemic, there is no agency that plans and coordinates psychological intervention for the country and her population. It would be worthwhile to consider involving psychiatrists and mental health professionals in the Task Force on COVID-19 to advise the government on mental health policies and psychological intervention. At this writing, the hospitals, polyclinics and SSA are working in silos to conduct psychological interventions in patients with little communication among them, leading to resource wastage and decreased efficacy of their interventions. It would be helpful to hospitals and SSA to align their goals and efforts by engaging each other in case discussions and training. Community health personnel can be trained to better identify and manage psychological distress in patients. Case discussions can promote seamless transfer of patient care across hospitals and community services. While patients with severe psychiatric morbidities will benefit from management in hospitals, mild to moderate cases or those who have recovered with treatment can be discharged to community services for continued management.
Past pandemics have provided Singapore valuable lessons on global responses to manage them. Consequently, the country is more medically prepared to deal with the COVID-19 outbreak with better medical infrastructure and technology and highly qualified health workers. However, it is crucial that we do not ignore the psychological impact the outbreak will have on individuals and society which can hamper their readiness to overcome the crisis, and the fact that the psychological ramifications can persist long after the pandemic has ended.

The outbreak of COVID-19 has highlighted the fragility of mental resilience and the need to have a nation-wide psychological intervention plan. We have suggested 6 strategies that local and overseas authorities could consider to improve their current plan. After their psychological defence is bolstered, countries will be equipped to succeed in their battle against COVID-19 and secure their future.

\section{REFERENCES}

1. Singapore Tourism Board. Monthly International Visitor Arrivals. Available at: https://www.stb.gov.sg/content/stb/en/statistics-andmarket-insights/tourism-statistics/international-visitorarrivals.html. Accessed on 22 February 2020.

2. Worldometer. COVID-19 Coronavirus Outbreak. Available at: https:// www.worldometers.info/coronavirus/.Accessed on 22 February 2020.

3. Ministry of Health, Singapore. Updates on COVID-19 (Coronavirus Disease 2019) Local Situation. Available at: https://www.moh.gov. sg/covid-19. Accessed on 22 February 2020.

4. Wang C, Pan R, Wan X, Tan Y, Xu L, Ho CS, et al. Immediate psychological responses and associated factors during the initial stage of the 2019 coronavirus disease (COVID-19) epidemic among the general population in China. Int J Environ Res Public Health 2020;17:1729.

5. Rodin P, Ghersetti M, Odén T. Disentangling rhetorical subarenas of public health crisis communication: a study of the 2014-2015 Ebola outbreak in the news media and social media in Sweden. J Contingencies Crisis Manag 2018;27:237-46.

6. Tang L, Bie B, Park SE, Zhi D. Social media and outbreaks of emerging infectious diseases: a systematic review of literature. Am J Infect Control 2018;46:962-72.

7. Hall RCW, Hall RCW, Chapman MJ. The 1995 Kikwit Ebola outbreak: lessons hospitals and physicians can apply to future viral epidemics. Gen Hosp Psychiatry 2008;30:446-52.

8. Tucci V, Moukaddam N, Meadows J, Shah S, Galwankar SC, Kapur GB. The forgotten plague: psychiatric manifestations of Ebola, Zika, and emerging infectious diseases. J Glob Infect Dis 2017;9:151-6.

9. Müller N. Infectious diseases and mental health. In: Sartorius N, Holt RIG, Maj M, editors. Comorbidity of Mental and Physical Disorders. Basel: S Karger AG; 2015. p. 99-113.

10. Sim K, Chan YH, Chong PN, Chua HC, Soon SW. Psychosocial and coping responses within the community health care setting towards a national outbreak of an infectious disease. J Psychosom Res 2010;68:195-202. 
11. Shear MK. Grief and mourning gone awry: pathway and course of complicated grief. Dialogues Clin Neurosci 2012;14:119-28.

12. Hawryluck L, Gold WL, Robinson S, Pogorski S, Galea S, Styra R. SARS control and psychological effects of quarantine, Toronto, Canada. Emerg Infect Dis 2004;10:1206-12.

13. Van Bortel T, Basnayake A, Wurie F, Jambai M, Koroma AS, Muana AT, et al. Psychosocial effects of an Ebola outbreak at individual, community and international levels. Bull World Health Organ 2016;94:210-4.

14. Leung GM, Lam TH, Ho LM, Ho SY, Chan BHY, Wong IOL, et al. The impact of community psychological responses on outbreak control for severe acute respiratory syndrome in Hong Kong. J Epidemiol Community Health 2003;57:857-63.

15. McAlonan GM, Lee AM, Cheung V, Cheung C, Tsang KWT, Sham $\mathrm{PC}$, et al. Immediate and sustained psychological impact of an emerging infectious disease outbreak on health care workers. Can J Psychiatry 2007;52:241-7.

16. Tiong WW, Koh GCH. Ethical considerations in the review of Singapore's H1N1 pandemic response framework in 2009. Ann Acad Med Singapore 2013;42:246-50.

17. Naushad VA, Bierens JJ, Nishan KP, Firjeeth CP, Mohammad OH, Maliyakkal AM, et al. A systematic review of the impact of disaster on the mental health of medical responders. Prehosp Disaster Med 2019;34:632-43.

18. Chan AOM, Chan YH. Psychological impact of the 2003 severe acute respiratory syndrome outbreak on health care workers in a medium size regional general hospital in Singapore. Occup Med (Lond) 2004;54:190-6.

19. National Health Committee, People's Republic of China. Guidelines of Psychological Crisis Intervention for COVID-19 Pneumonia. Available at: http://www.nhc.gov.cn/jkj/s3577/202001/6adc08b966 594253b2b791be5c3b9467. Accessed on 24 February 2020.

20. Lim GY, Tam WW, Lu Y, Ho CS, Zhang MW, Ho RC. Prevalence of depression in the community from 30 countries between 1994 and 2014. Sci Rep 2018;8:2861.
21. Albert PR. Why is depression more prevalent in women? J Psychiatry Neurosci 2015;40:219-21.

22. Chen N, Zhou M, Dong X, Qu J, Gong F, Han Y, et al. Epidemiological and clinical characteristics of 99 cases of 2019 novel coronavirus pneumonia in Wuhan, China: a descriptive study. Lancet 2020;395:507-13

23. Ho RC, Zhang MW, Ho CS, Pan F, Lu Y, Sharma VK. Impact of 2013 South Asian haze crisis: study of physical and psychological symptoms and perceived dangerousness of pollution level. BMC Psychiatry 2014;414:81.

24. Zhang MWB, Ho CSH, Fang P, Lu Y, Ho RCM. Methodology of developing a smartphone application for crisis research and its clinical application. Technol Health Care 2014;22:547-59.

25. Maunder RG, Lancee WJ, Balderson KE, Bennett JP, Borgundvaag B, Evans S, et al. Long-term psychological and occupational effects of providing hospital healthcare during SARS outbreak. Emerg Infect Dis 2006;12:1924-32.

26. Carlson LE. Mindfulness-based interventions for physical conditions: a narrative review evaluating levels of evidence. ISRN Psychiatry 2012: 651583 .

27. Low JGH, Wilder-Smith A. Infectious respiratory illnesses and their impact on healthcare workers: a review. Ann Acad Med Singapore 2005;34:105-10.

28. Chua SE, Cheung V, Cheung C, McAlonan GM, Wong JWS, Cheung EPT, et al. Psychological effects of the SARS outbreak in Hong Kong on high-risk health care workers. Can J Psychiatry 2004;49:391-3.

29. Deurenberg-Yap M, Foo LL, Low YY, Chan SP, Vijaya K, Lee M. The Singaporean response to the SARS outbreak: knowledge sufficiency versus public trust. Health Promot Int 2005;20:320-6.

30. O'Shea BA, Watson DG, Brown GDA, Fincher CL. Infectious disease prevalence, not race exposure, predicts both implicit and explicit racial prejudice across the United States. Soc Psychol Pers Sci 2019:doi.org/10.1177/1948550619862319. 